\title{
ENSEÑANZA DE LA HISTORIA, ANÁLISIS DE LIBROS DE TEXTO Y CONSTRUCCIÓN DE IDENTIDADES COLECTIVAS*
}

\author{
History teaching, analysis of textbooks and the making of collective \\ identities
}

Pedro Miralles Martínez ${ }^{\varnothing}$ y Cosme J. Gómez Carrasco ${ }^{\S}$

\section{LA HISTORIA. ENTRE LA CONSTRUCCIÓN DE IDENTIDADES Y LA EDUCACIÓN CIUDADANA. EL PAPEL DE LOS LIBROS DE TEXTO EN LA REPRODUCCIÓN DE UN MODELO DE EDUCACIÓN HISTÓRICA}

Los debates en las dos últimas décadas sobre la enseñanza de la historia en el ámbito académico han girado en torno a la dualidad entre competencias y conocimientos conceptuales; entre el papel que deben tener los contenidos sustantivos, las habilidades relacionadas con la práctica del historiador y las competencias educativas. ${ }^{1}$ Estos debates, que han surgido en diferentes contextos sociales, territoriales y políticos, tienen un elemento común: la pugna entre los dos tipos de lógica que han articulado la enseñanza de la historia escolar desde que surgieron los Estados liberales. Esta dinámica reconoce la racionalidad crítica de la Ilustración (que en la enseñanza de la historia responde a la necesidad de educar

\footnotetext{
* Este trabajo es resultado del proyecto de investigación «La evaluación de las competencias y el desarrollo de capacidades cognitivas sobre historia en Educación Secundaria Obligatoria» (EDU201565621-C3-2-R), subvencionado por el Ministerio de Economía y Competitividad español.

${ }^{\circ}$ Departamento de Didáctica de las Ciencias Matemáticas y Sociales. Facultad de Educación. Universidad de Murcia. Campus Universitario de Espinardo s/n. 30100, Murcia. España. pedromir@um.e $\S$ Departamento de Didáctica de las Ciencias Matemáticas y Sociales. Facultad de Educación. Universidad de Murcia. Campus Universitario de Espinardo s/n. 30100, Murcia. España. cjgomez@um.es ${ }^{1}$ Penney Clark, New possibilities for the past. Shaping history education in Canada (Vancouver \& Toronto: UBC Press, 2011); Cosme J. Gómez, Ortuño, Jorge Ortuño y Sebastián Molina, «Aprender a pensar históricamente. Retos para la historia en el siglo XXI», Tempo e Argumento, 6 (11), (2014): 1-25; Stephane Lévesque, Thinking Historically. Educating Students for the 21th Century, (Toronto: University of Toronto Press, 2008); Bruce VanSledright, «Narratives of Nation-State, Historical Knowledge and School History Education», Review of Research in Education, 32 (1), (2008): 109-146.
}

Cómo citar este artículo: Miralles Martínez, Pedro y Gómez Carrasco, Cosme J. «Enseñanza de la historia, análisis de libros de texto y construcción de identidades colectivas», Historia y Memoria de la Educación, 6 (2017): 9-28. 
en valores cívicos y sociales, pero también en aspectos cognitivos de la disciplina histórica, su racionalidad científica, etc.), y la emotividad identitaria del Romanticismo (que en la enseñanza de la historia responde a la necesidad de que el alumnado se impregne de las hazañas patrias y de contenidos que permitan cumplir con objetivos de tipo identitario). ${ }^{2}$ Este último elemento está muy relacionado con la construcción de la memoria colectiva. Gracias a nuestra naturaleza humana tenemos conciencia del tiempo, y a menudo utilizamos esa conciencia a través de constructos temporales relacionados con nuestra identidad, origen, memoria, o la relación entre el pasado, presente y futuro. ${ }^{3}$ Una memoria colectiva que, en palabras de Le Goff, ${ }^{4}$ a los ojos de los historiadores aparece como mítica, deformada y anacrónica. Pero que se nutre del conocimiento escolar, de los medios de comunicación y de otros medios informales de conocimiento.

Identidad y ciudadanía son dos conceptos en torno a los que han girado las múltiples funciones de la historia, lo que denota una ciencia compleja que a veces ha sido sometida a los intereses del poder. De hecho, como indican Miralles y Alfageme, ${ }^{5}$ los conceptos de ciudadanía e identidad han estado ligados a la formación de los Estados-nación, y muy vinculados a la función instructiva de la historia y a la creación de señas de identidad nacional. ${ }^{6}$ El conocimiento histórico es hijo de la memoria, pero sin duda esta es subjetiva y debe ser "objetivada" por el aprendizaje de los métodos del historiador. ${ }^{7}$ Evidentemente memoria no es historia

\footnotetext{
${ }^{2}$ Mario Carretero, Alberto Rosa y María Fernanda González, «Enseñar historia en tiempos de memoria», en Enseñanza de la historia y memoria colectiva, comps. Mario Carretero, Alberto Rosa y María Fernanda González (Buenos Aires: Editorial Paidós, 2006), 11-36.

${ }^{3}$ Arthur Chapman, «Historical interpretations», en Debates in History Teaching, ed. Ian Davies (London-New York: Routledge, 2011); Paul Ricoeur, History, Memory, Forgetting (Chicago \& London: University of Chicago Press, 2004); Jörn Rüsen, History: Narration, Interpretation, Orientation (New York: Berghahn, 2005).

${ }^{4}$ Jacques Le Goff, Histoire et memoire (Paris: Galimard, 1988).

${ }^{5}$ Pedro Miralles y María Begoña Alfageme, «Educación, identidad y ciudadanía en un mundo globalizado y posmoderno», Educatio siglo XXI, 31 (1), (2013): 11-12.

${ }^{6}$ Keith C. Barton, «Historia e identidad: el reto de los investigadores pedagógicos», en Metodología de la investigación en Didáctica de las Ciencias Sociales (Zaragoza: Instituto Fernando el Católico-AUPDCS, 2010), 13-28.

${ }^{7}$ Arthur Chapman, «Taking the perspective of the other seriously? Understanding historical argument», Educar em Revista, 42, (2011): 95-106; Joaquim Prats y Joan Santacana, "¿Por qué y para qué enseñar historia?», en Didáctica de la Geografía y la Historia, coord. Joaquim Prats (Barcelona: Graó 2011), 13-29.
} 
y no hay que caer en el "presentismo». ${ }^{8}$ Se trataría de transformar la memoria que construimos las personas, como almacén de datos, hechos y emociones, en historia, como archivo de representaciones significativas reelaboradas consciente y críticamente. ${ }^{9}$ Nuestra propia identidad depende de nuestra memoria, algo que es válido tanto para los sujetos individuales, como para los grupos y las instituciones. Tanto en la investigación como en la enseñanza de la historia la memoria debe ser «deconstruida» para convertirla en historia. Ese proceso que une memoria, historia e identidades se ha usado habitualmente por el poder a través del arte, la literatura, el cine o los medios de comunicación, para trasladar su mensaje y convertirlo en verdad socialmente aceptada. ${ }^{10} \mathrm{Y}$ en este caso los libros de texto son un «artefacto cultural» clave para comprender las propuestas que provienen del poder para construir su concepto de identidad social, cultural y política.

No cabe duda que la contraposición de ambas visiones de la enseñanza de la historia (identidad y ciudadanía) y de su función social son casi antagónicas, o al menos de muy difícil complementación. Entre otras razones porque una forma de enseñar historia fomenta, sobre todo, la creación de identidades colectivas excluyentes al tener como eje discursivo el relato nacional, cuando no étnico o cultural basado en subrayar las diferencias existentes entre el «nosotros» $y$ «los otros». Mientras que el otro enfoque, a través de la potenciación del espíritu crítico, pretende ayudar a la construcción de identidades múltiples o inclusivas (las fidelidades concéntricas de las que nos ha hablado López Facal). ${ }^{11}$ Unas identidades en la que se muestren las características que tienen los distintos

\footnotetext{
8 Joaquim Prats, «Memoria histórica "versus" historia enseñada», Íber. Didáctica de las Ciencias Sociales, Geografía e Historia, 55, (2008): 5-6; Mario Carretero y Marcelo Borrelli, «Memoria y enseñanza de la historia en un mundo global. Problemas y desafíos», Íber. Didáctica de las Ciencias Sociales, Geografía e Historia, 55, (2008): 22-25.

${ }^{9}$ Pedro Miralles, «La didáctica de la historia en España: retos para una educación de la ciudadanía, en L'educazione alla cittadinanza europea e la formazione degli insegnanti. Un progetto educativo per la «Strategia di Lisbona», eds. Rosa M. Ávila, Beatrice Borghi e Ivo Mattozzi (Bologna: Pàtron Editore, 2009), vol. I, 265.

${ }^{10}$ Alberto Rosa, «Memoria, historia e identidad. Una reflexión sobre el papel de la enseñanza de la historia en el desarrollo de la ciudadanía», en Aprender y pensar la historia, comps. Mario Carretero y James Voss (Madrid: Amorrortu, 2004), 47-70.

${ }^{11}$ Ramón López Facal, «Identidades posnacionales y enseñanza», Íber. Didáctica de las Ciencias Sociales, Geografía e Historia, 47, (2006): 54-63.
} 
colectivos que componen una sociedad para dar a conocer los rasgos o elementos compartidos. ${ }^{12}$

Los análisis y las propuestas de Rüsen señalan la importancia de hacer de la historia un conocimiento útil para orientar éticamente los comportamientos personales. ${ }^{13}$ La historia, por tanto, no es una disciplina sólo para eruditos y tampoco es un saber estático, sino que debe entenderse en permanente construcción y con un importante potencial educativo en la formación como ciudadanos.

Desde comienzos del dos mil, se han extendido por toda la Unión Europea las recomendaciones de la OCDE de explicitar una formación de la ciudadanía responsable, activa y autónoma dentro de la educación básica del alumnado. Con esa intención la educación cívica forma parte del currículo de todos los países de la Unión Europea. ${ }^{14}$ En España desde el currículo educativo de 2006 (LOE) se incorporaron las competencias como elementos clave en la enseñanza de las diferentes materias. Entre estas competencias se encuentra la "Competencia social y ciudadana», que después con la LOMCE se ha denominado como «Competencias sociales y cívicas». A pesar de que la introducción de este nuevo concepto educativo no ha sido fácil, creando toda una serie de polémicas y dudas respecto a su origen, contenidos y saberes, ${ }^{15}$ en ambos decretos se insiste en que la finalidad última de esta competencia es «formar ciudadanos y ciudadanas capaces de saber convivir democráticamente con los demás, de participar en la vida social, laboral, cultural y política de su mundo, intentando mejorarla». ${ }^{16} \mathrm{El}$ papel de la historia y otras ciencias sociales en el desarrollo de esta competencia es clave. Como indica López Facal, ${ }^{17}$ ser competente implica saber interpretar el medio en el que el alumno

\footnotetext{
${ }^{12}$ Ramón López Facal, «Enseñar historia en convivencia plurinacional», Gerónimo de Ustariz, 17-18 (2002): 49-57.

${ }^{13}$ Jörn Rüsen, History: Narration, Interpretation, Orientation (New York: Berghahn, 2005).

${ }^{14}$ Pedro Miralles, Sebastián Molina y Jorge Ortuño, La importancia de la historiografía en la enseñanza de la historia (Granada: GEU, 2011).

${ }^{15}$ José Monteagudo y José Luis Villa, «La evaluación de las competencias básicas en la materia de Historia de $4 .^{\circ}$ de ESO de la Región de Murcia», en La evaluación en el proceso de enseñanza y aprendizaje de las ciencias sociales, eds. Pedro Miralles Martínez, Sebastián Molina Puche y Antoni Santisteban Fernández (Murcia: AUPDCS, 2011), vol. I, 317-326.

${ }^{16}$ Joan Pagès, «Competencia social y ciudadana», Aula de innovación educativa, 187 (7), (2009): 7-11.

${ }^{17}$ Ramón López Facal, «Competencias y enseñanza de las ciencias sociales», Íber. Didáctica de las Ciencias Sociales, Geografía e Historia, 74 (2013): 5-8.
} 
interactúa, saber proponer alternativas, ser capaz de argumentar. Estas operaciones necesitan de un conocimiento sobre cómo es y cómo funciona la sociedad, cómo se han ido generando y modificando las relaciones humanas a lo largo del tiempo, qué consecuencias han tenido y tienen las acciones que realizan las personas y los colectivos.

En respuesta a estas propuestas curriculares, que hacen énfasis en la formación de unos valores cívicos y sociales basados en la participación y la reflexión crítica, muchas de las investigaciones sobre la enseñanza de la historia han puesto en valor esas posibilidades formativas para crear ciudadanos activos y participativos, con un enfoque muy ligado a las ciencias sociales y cercano al discurso crítico heredado de la Ilustración. ${ }^{18}$ En estos trabajos se defiende la necesidad de un diálogo entre la historia, las ciencias sociales y la educación para la ciudadanía. Ésta debe incorporar la dimensión social, espacial y temporal para evitar la descontextualización y los estereotipos. Las ciencias sociales deben revisar la vieja idea de ciudadanía para avanzar hacia una ciudadanía cosmopolita que compatibilice identidades plurales. ${ }^{19}$ Los trabajos de García y De Alba en España, ${ }^{20}$ de Starkey en Inglaterra ${ }^{21}$ o de Schugurensky en el continente americano ${ }^{22}$ son ejemplos de la preocupación por una educación para la ciudadanía, los derechos humanos y la participación democrática a la hora de abordar la enseñanza de las ciencias sociales.

\footnotetext{
${ }^{18}$ Mario Carretero y Miriam Kriger, «¿Forjar patriotas o educar cosmopolitas? El pasado y el presente de la historia escolar en un mundo global», en Aprender y pensar la historia, comps. Mario Carretero y James Voss (Buenos Aires: Amorrortu, 2004), 71-98.

${ }^{19}$ Xose M. Armas y Ramón López Facal, «Ciencias sociales y educación para la ciudadanía. Un diálogo necesario», Íber. Didáctica de las Ciencias Sociales, Geografía e Historia, 71, (2012): 84-92.

${ }^{20}$ Francisco García Pérez y Nicolás de Alba, «Educar para la participación ciudadana. Análisis de las dificultades del profesorado a partir de la experiencia del programa Parlamento Joven», en L'educazione alla cittadinanza europea e la formazione degli insegnanti. Un progetto educativo per la «Strategia di Lisbona», eds. Rosa M. Ávila, Beatrice Borghi e Ivo Mattozzi (Bolonia: Pàtron Editore, 2009), vol. 1, 515-521.

${ }^{21}$ Hugh Starkey, "Citizenship, education and global spaces», Language and Intercultural Communciation, 11 (2), (2011): 75-79; Hugh Starkey «Human Rights and Education», en Encyclopedia of Diversity in Education, ed. James Banks (London: SAGE, 2012), 1115-1118.

${ }^{22}$ David Schugurensky, "Civic engagement and participatory governance», en Civic Engagement: 100th Arizona Town Hall, ed. R. Kelly (Phoenix: Arizona Town Hall, 2012); Elisabeth Pinnington \& David Schugurensky (eds.), Learning citizenship by practicing democracy: international initiatives and perspectives (Newcastle upon Tyne: Cambridge Scholarls Publishing, 2010).
} 
Este enfoque de enseñanza, que incide más en la práctica social que en los procesos cognitivos individuales, ha sido definido por Barton y Levstik como perspectiva socio-cultural de la educación histórica. ${ }^{23}$ Desde esta perspectiva la enseñanza de la historia se plantea desde un contexto principalmente social, incidiendo en los protagonistas de los procesos históricos, la agencia histórica, o el propósito y motivaciones que hay detrás de los acontecimientos. Una visión humanística de la enseñanza de la historia que intenta promover la educación ciudadana a través del razonamiento sobre los acontecimientos sociales en el pasado; una visión amplia de la humanidad; y una reflexión de conjunto sobre lo que significa el bien común, The common good. ${ }^{24}$ Estos planteamientos pasan inevitablemente por la enseñanza de la historia desde un enfoque crítico y reflexivo. ${ }^{25}$

Unas propuestas que han sido puestas en valor también desde el ámbito francófono. ${ }^{26}$ Heimberg insistió en que la historia permite construir una mirada lúcida y un sentido crítico sobre el mundo al evocar todas las maneras en que los hombres se han organizado colectivamente y cómo han hecho frente a sus problemas, conflictos e intereses. ${ }^{27}$ Ese potencial educativo no puede perderse en una maraña de datos, fechas y hechos. El objetivo principal de la historia debe ser hacer reflexionar sobre esos procesos históricos. Una visión que comparte con Audigier, ${ }^{28}$ que indicó que la enseñanza de la historia desde la perspectiva de la educación cívica está muy relacionada con el proyecto y las esperanzas de futuro, y

\footnotetext{
${ }^{23}$ Keith Barton y Linda Levstik, Teaching History for the Common Good (New Jersey: Lawrence Erlbau, 2004).

${ }^{24}$ Barton y Levstik, Teaching History; Peter Seixas, «The Community of Inquiry as a Basis for Knowledge and Learning: The Case of History», American Educational Research Journal, 30 (2), (1993): 305-324.

${ }^{25}$ Rosa Hervás y Pedro Miralles, «Nuevas formas de enseñar a pensar. El desarrollo del pensamiento crítico en la enseñanza de las ciencias sociales», Íber. Didáctica de las Ciencias Sociales, Geografía e Historia, 42, (2004): 98-99.

${ }^{26}$ François Audigier y Nicole Tutiaux-Guillon, Regards sur l'histoire, la géographie et l'éducation civique à l'école primaire (Lyon: INRP, 2004); Franck Thénard-Duvivier (coord.), L'enseignement en histoire et géographie (Paris: ADAPT, 2008).

${ }^{27}$ Charles Heimberg, «Pour une éducation aux citoyennetés ouverte sur le monde», en L'école du citoyen. Revue Nationale du Réseau CNDP-CRDP pour l'enseignement de l'histoire et de la géographie, 7, (1999): 163-171; Charles Heimberg, «L'enseignement de l'histoire dans un pays d'immigration: la Suisse, en Pensar históricamente en tiempos de globalización (Santiago de Compostela: USC, 2011), 21-35.

${ }^{28}$ François Audigier, L'éducation à la citoyenneté (Paris: INRP, 1999).
} 
los valores dominantes de participación democrática, libertad y espíritu crítico.

Así pues, en un mundo cada vez más globalizado, en el que las sociedades están muy alejadas de esa uniformidad étnica, cultural, lingüística y religiosa que propugnaban los Estados-nación decimonónicos, la que podríamos denominar como "visión tradicional» de la enseñanza de la historia debiera tener, cada vez, menor cabida en las aulas. En palabras de Miralles y Alfageme, ${ }^{29}$ tratar sobre estos temas (identidad y ciudadanía) nos remite a conceptos fundamentales en la teoría y el pensamiento social actual, como son globalización y posmodernidad. Para Bauman, ${ }^{30}$ sociólogo de la «modernidad líquida», las actuales circunstancias del capitalismo global han ocasionado una nueva precariedad que trata de sobreponerse por medio de dos alternativas: la reconstrucción de los vínculos colectivos por medio de nuevas «comunidades de elección»; y la creación de un sentimiento comunitario por medio de las nuevas políticas de la diferencia, que aceptan el multiculturalismo y la política del reconocimiento. ${ }^{31}$ Globalización y posmodernidad están en el origen de un fenómeno importante de reseñar: el surgimiento de identidades múltiples. La compleja composición de la sociedad actual demanda de las propuestas de enseñanza de la historia la aceptación de la alteridad, el reconocimiento de la diversidad de identidades, y el derecho de los individuos a demandar su propia identidad. ${ }^{32}$

Sin embargo, y a pesar de los trabajos anteriores que pretenden impulsar una educación histórica basada en valores cívicos y en la participación democrática, los estudios realizados en las últimas décadas para intentar determinar cómo se enseña la historia, qué contenidos predominan en las aulas, y con qué finalidad tanto en España ${ }^{33}$ como en otros

\footnotetext{
${ }^{29}$ Miralles y Alfageme, «Educación, identidad y ciudadanía en un mundo globalizado y posmoderno", 13-15.

${ }^{30}$ Zigmunt Bauman, Modernidad líquida (Buenos Aires: Corregidor, 2003).

${ }^{31}$ Pedro Miralles, Joaquim Prats y Mercé Tatjer, «Conocimientos y concepciones de estudiantes españoles y latinoamericanos de Educación Secundaria Obligatoria sobre las independencias políticas americanas», Scripta Nova. Revista Electrónica de Geografía y Ciencias Sociales, XVI (418), (2012): 1-2; Miralles y Alfageme, «Educación, identidad y ciudadanía», 13-14.

${ }^{32}$ Miralles, Prats y Tatjer, «Conocimientos y concepciones de estudiantes», 11-12.

${ }^{33}$ Cosme J. Gómez y Pedro Miralles, «Los contenidos de ciencias sociales y las capacidades cognitivas en los exámenes de tercer ciclo de Educación Primaria ¿Una evaluación en competencias?», Revista Complutense de Educación, 24 (1), (2013): 91-121; Cosme J. Gómez y Pedro Miralles, «¿Pensar
} 
países occidentales, ${ }^{34}$ han demostrado que la realidad es bien distinta: la historia (al menos desde las propuestas curriculares oficiales y en las rutinas dominantes del aula) sigue manteniendo un discurso muy descriptivo, lineal y acrítico, y se sigue instrumentalizando para reforzar la identidad nacional.

En efecto, la historia ha sido una de las disciplinas que más han contribuido a conformar la visión sobre la identidad social y política de las naciones. ${ }^{35}$ Las raíces intelectuales del Romanticismo están muy filtradas en la enseñanza de la historia en la escuela, y muy relacionadas con el surgimiento de los Estados-nación. Hobsbawn, que llegó a negar la antigüedad de las naciones, defendía que eran« artefactos inventados» e imaginados. ${ }^{36}$

El uso del pasado y la instrumentalización de la tradición por parte estas naciones en su proceso de legitimación ha sido puesto en valor por autores como Álvarez Junco, ${ }^{37}$ que ha insistido en la estrecha relación que ésta tiene con el nacionalismo y la construcción de identidades nacionales. Hobsbawn hablaba de «la invención de la tradición» para explicar esa relación entre el nacionalismo y el uso de la historia como instrumento de legitimación. Este autor indicó que el término de «tradición inventada» incluye tanto las tradiciones que realmente son inventadas, como aquéllas que emergen de una manera rápida y se consolidan como «tradiciones» aunque no lo sean. Hobsbawn pone como ejemplo de estas últimas la

históricamente o memorizar el pasado? La evaluación de los contenidos históricos en la educación obligatoria en España», Revista de Estudios Sociales, 52, (2015): 52-68; Ramón López Facal, «Nacionalismos y europeísmos en los libros de texto: identificación e identidad nacional». Clío y asociados: la historia enseñada, 14, (2010): 9-13; Jorge Sáiz, «Educación histórica y narrativa nacional» (Tesis doctoral, Universidad de Valencia, 2015).

${ }^{34}$ Isabel Barca, O pensamento histórico dos joves (Braga: Universidade do Minho, 2000); Keith C. Barton, "Historia e identidad: el reto de los investigadores pedagógicos», en Metodología de la investigación en Didáctica de las Ciencias Sociales (Zaragoza: Instituto Fernando el Católico-AUPDCS, 2010), 13-28; Charles Heimberg, «L'apport de l'histoire à l'éducation à la citoyenneté», en Vivre la democratie, apprende la democratie (Berne: SER, 2005), 45-50.

${ }^{35}$ Joaquim Prats, «En defensa de la historia como materia educativa», Tejuelo. Didáctica de la Lengua y la Literatura, 9, (2010): 8-17.

${ }^{36}$ Eric J. Hobsbawm, «Introducción: la invención de la tradición», en La invención de la tradición, coords. Eric J. y Terence Rangers (Barcelona: Crítica, 2002), 7-21.

${ }^{37}$ José Álvarez Junco, Dioses útiles. Naciones y nacionalismos (Barcelona: Galaxia Gutenberg, 2016); José Álvarez Junco (coord.), Las historias de España. Visiones del pasado y construcción de identidad (Madrid: Crítica-Marcial Pons, 2013). 
pompa que rodea a las manifestaciones ceremoniales de la monarquía británica. ${ }^{38}$ Unas ceremonias que tienen su origen a finales del siglo XIX y principios del siglo xx, pero que reclaman una antigüedad que no es real. Estas tradiciones inventadas establecen o simbolizan cohesión social o pertenencia al grupo, ya sean comunidades reales o artificiales; legitiman instituciones, estatus o relaciones de autoridad; y tienen como primer objetivo la socialización, la inculcación de creencias o sistemas de valores. ${ }^{39}$

Si la historia y los historiadores tienen la capacidad de jerarquizar el pasado, los poderes del Estado siempre han tenido claro la necesidad de controlar o condicionar ese poder cultural en ese afán de homogeneizar la identidad. ${ }^{40} \mathrm{Y}$ la geografía y la historia han sido dos materias educativas clave que han permitido difundir el discurso que legitima la nación frente a otras realidades o identidades. Carretero, Castorina, Van Alphen, Sarti y Barreiro realizaron un interesante análisis sobre los objetivos de la enseñanza de la historia relacionados con esta función identitaria, y que solían plasmarse en los libros de texto. ${ }^{41}$ Según estos autores, la influencia del Romanticismo en la enseñanza de la historia se manifiesta a través de tres cuestiones centrales: una valoración positiva del pasado y presente del propio grupo social, tanto en el ámbito local como nacional; la valoración positiva de la historia política del país; y la identificación con los acontecimientos del pasado, personajes y héroes nacionales.

Aunque algunos estudios consideran que el problema puede estar en la formación inicial del profesorado, que en casos como el español se encuentra muy centrada en la disciplina histórica y muy poco en la parte más didáctica o pedagógica, lo que lleva a que muchos de los nuevos profesores acaben reproduciendo los métodos y modos de enseñanza de sus propios «maestros»; ${ }^{42}$ buena parte de los estudios inciden que el prin-

\footnotetext{
${ }^{38}$ Hobsbawm, «Introducción: la invención de la tradición», 7-21.

${ }^{39}$ Hobsbawm, «Introducción: la invención de la tradición», 7-21.

40 Juan Sisinio Pérez-Garzón, «Usos y abusos de la historia», Gerónimo de Ustariz, 17-18 (2002): 11-24, y «¿Por qué enseñamos geografía e historia? ¿Es tarea educativa la construcción de identidades?», Historia de la educación, 27, (2008): 37-55.

${ }^{41}$ Mario Carretero, José Antonio Castorina, María Sarti, Floor Van Alphen y Alicia Barreiro, «La construcción del conocimiento histórico», Propuesta Educativa, 39 (1), (2013): 13-23.

${ }^{42}$ Cosme J. Gómez, Raimundo A. Rodríguez y Ana B. Mirete, «Relación entre metodología docente y uso de recursos de innovación en la enseñanza de la historia. Un análisis a través de los recuerdos de los maestros en formación», Clío. History and History Teaching, 42 (2016).
} 
cipal problema se encuentra en los currículos, ${ }^{43}$ y en la principal herramienta con la que cuentan los docentes: los manuales escolares. ${ }^{44}$

Las narrativas que se divulgan en los libros de texto son de gran importancia para comprender la construcción del pasado desde los poderes públicos. En opinión de Topolsky, ${ }^{45}$ las narrativas históricas deben ser el principal foco de interés en la actividad didáctica de la enseñanza de la historia. Varios autores han analizado los tópicos y estereotipos identitarios en las narrativas históricas, tanto oficialistas como las que producen los estudiantes. ${ }^{46} \mathrm{El}$ análisis de estas narrativas permite apreciar lo que Chartier definió como «la brecha existente entre el pasado y su representación, entre lo que fue y no es más, y las construcciones narrativas que se proponen ocupar el lugar de ese pasado». ${ }^{47} \mathrm{El}$ relato histórico que muestran los exámenes y los libros de texto está basado en una narración lineal y simple del pasado, que además se exige conocer a través de la memorización de datos, sin aplicación de conocimientos y sin valoración crítica. ${ }^{48}$ Es un discurso muy relacionado con esos objetivos románticos de la historia comentados anteriormente. La enseñanza de la historia,

${ }^{43}$ Heimberg, «L’enseignement de l’histoire», 21-35; Pérez-Garzón, «¿Por qué enseñamos geografía e historia?», 37-55; Francisco Rodríguez Lestegás, «La construcción de identidades en la sociedad actual: un desafío para la educación y para la democracia. Revista Española de Pedagogía, 239 (2008): 85-102.

${ }^{44}$ Cosme J. Gómez, Ramón Cózar y Pedro Miralles, «La enseñanza de la historia y el análisis de libros de texto. Construcción de identidades y desarrollo de competencias», Ensayos. Revista de la Facultad de Educación de Albacete, 29 (1), (2014): 1-25; Ramón López Facal, «Nacionalismos y europeísmos en los libros de texto: identificación e identidad nacional», Clío y asociados: la historia enseñada, 14, (2010): 9-13; Jason Nicholls, "Methods in School Textbooks Research», International Journal of Historical Learning. Teaching and Research, 3 (1), (2003); Stephen J. Thornton, «What is history in US history textbooks?», en School history textbooks across cultures: International debates and perspectives ed. Jason Nicholls (Oxford, UK: Symposiumbooks, 2006), 15-25; Rafael Valls, La enseñanza de la Historia y textos escolares (Buenos Aires: Zorzal, 2008).

45 Jerzy Topolsky, «La estructura de las narrativas históricas y la enseñanza de la historia», en Aprender y pensar la historia, comp. Mario Carretero y James Voss (Buenos Aires: Amorrortu, 2004), 101-120.

${ }^{46}$ Barton y Levstik, Teaching History; Grant Bage, Narrative Matters. Teaching and Learning History through Story (New York: Routledge, 1999); Mario Carretero, "Comprensión y aprendizaje de la historia», en Enseñanza y aprendizaje de la historia en la educación básica (México: Secretaría de Educación Pública, 2011), 69-104; Chauncey Monte-Sano, «Disciplinary literacy in history: An exploration of the historical nature of adolescents' writing», The Journal of the Learning Sciences, 19 (4), (2010): 539-568.

${ }^{47}$ Roger Chartier, La historia o la lectura del tiempo (Madrid: Gedisa, 2007), 22.

${ }^{48}$ Cosme J. Gómez, «Pensamiento histórico y contenidos disciplinares en los libros de texto. Un análisis exploratorio de la Edad Moderna en 2. ${ }^{\circ}$ de ESO», Ensayos. Revista de la Facultad de Educación de Albacete, 29 (1), (2014): 131-158. 
desde esta perspectiva, se fundamenta en la trasmisión de una memoria colectiva que ensalza el valor de los padres fundacionales y de los héroes de la nación. ${ }^{49}$

El libro de texto es probablemente la narrativa histórica más leída por el conjunto de la sociedad, y quizás el único relato sobre historia que utilicen a lo largo de su vida junto con otros medios informales de conocimiento (medios de comunicación, museos, otros centros de interés histórico, etc.). Por ello tiene tanta importancia el análisis de ese relato, de esa narrativa, que ha creado tantas controversias por su uso (y abuso) en el ámbito educativo. ${ }^{50}$

\section{DOSSIER «REPRESENTACIONES SOCIALES Y CONSTRUCCIÓN DE IDENTIDADES COLECTIVAS EN LOS LIBROS DE TEXTO DE HISTORIA. UN ESTUDIO COMPARATIVO EUROPA-AMÉRICA LATINA»}

La preocupación sobre el papel de los libros de texto de historia en la creación de identidades colectivas justifica esta propuesta que integra diferentes perspectivas de investigación (desde la historia de la educación a investigaciones sobre educación histórica y didáctica de las ciencias sociales), una gran diversidad de realidades territoriales (Inglaterra, Suecia, Portugal, España y Brasil), e institucionales (diez universidades distintas).

La mayor parte de los trabajos combinan estudios diacrónicos de libros de texto en comparación con los actuales. Desde una perspectiva más cercana a la historia de la educación, el trabajo de M. Teresa Santos Da Cunha, de la Universidad de Santa Catarina (Brasil) analiza las biografías de personajes históricos en los manuales escolares entre 1950-1970. Estas biografías laudatorias de los llamados "héroes de la historia nacional» se desarrollaron en forma de una hagiografía cívica y patriótica, que buscaban reforzar los principios de la República. La presencia de estos personajes heroicos en los textos se realizaba en una

\footnotetext{
${ }^{49}$ Bruce VanSledright, The Challenge of Rethinking History Education. On Practice, Theories, and Policy (New York: Routledge, 2011).

${ }^{50}$ Stuart J. Foster \& Keith A. Crawford (eds.), What Shall We Tell the Children? International Perspectives on School History Textbooks (Greenwich, CT: Information Age Publishing, 2006).
} 
comunidad simbólica a través de sus acciones, representadas como signos de lo que la autora denomina «brasilidad». En este artículo la autora analiza los libros de texto como artefactos culturales de una época, utilizados como vehículos de valores morales que tenían que asimilar los estudiantes.

Desde una perspectiva similar, Marcia Santos (Universidad de Santa Catarina, Brasil) analiza las perspectivas sobre la educación cívica que fueron representadas en los libros de texto de Educación Moral y Cívica en la década de 1970. Para ello ha utilizado tres manuales de los más utilizados en Florianópolis, capital de Santa Catarina. La investigación se ha basado en la identificación de las huellas que hicieron evidentes los contenidos que estaban destinados a formar a los ciudadanos de ese momento, su relación con la prescripción y civismo representado en los contenidos de los libros de texto. Como marco teórico, este artículo ha partido del concepto de representaciones sociales formuladas desde la historia cultural desde la tradición de Roger Chartier.

Gloria Solé (Universidad Do Minho, Portugal) analiza las representaciones sociales de los manuales escolares de Educación Primaria en Portugal desde una perspectiva también diacrónica. Este artículo parte desde un enfoque teórico que considera los libros de texto como mediadores entre los programas, escuelas historiográficas, políticas educativas, sistemas normativos educativos y diferentes perspectivas de enseñanza. Unos manuales que reflejan necesariamente las representaciones sociales de un momento dado en un contexto dado, y que fomentan la construcción de identidades sociales y políticas, que puede ser más o menos homogéneas. En este trabajo se analizan las representaciones sociales (mujer, hombre, belleza, etc.), así como la construcción de las identidades múltiples (sociales, políticos, culturales) desde principios del siglo Xx hasta la actualidad (Primera República, Estado Novo, después de la Revolución del 25 de abril de 1974, las décadas de 1980 y 1990 y el año actual).

El artículo de Ingmarie Danielsson Malmros (Universidad de Ümea, Suecia) estudia las narrativas presentes en los libros de texto. Este trabajo pone el foco sobre las narrativas que las sociedades eligen transmitir a las nuevas generaciones como interpretaciones y construcciones de la realidad. Para ello la autora investiga el significado que transmiten narrativas sobre Suecia en los libros de texto de historia publicados entre 1931 
y 2009. El objetivo ha sido conocer cómo esas narrativas se han transferido y cambiado con el tiempo y qué factores pueden haber influido en estos cambios. Epistemológicamente, este artículo presupone que los seres humanos construyen relatos de varias maneras para construir identidades. Sin embargo, estas narrativas no son vistas como reflexiones precisas de una realidad pasada, sino como medios para crear orden en el mundo mediante la incorporación de eventos en estructuras temporales.

Muy relacionado con el anterior artículo es el que presenta Jorge Sáiz, de la Universidad de Valencia. Este trabajo analiza los cambios y continuidades en la historia escolar de un contenido relevante de la narrativa histórica española tradicional: la génesis de España con los Reyes Católicos y la formación y auge de un imperio español con los Austrias. Se examinan dos fuentes: libros de texto de Bachillerato de las editoriales mayoritarias entre 1975 y 2015; y narrativas históricas de estudiantes al finalizar educación secundaria y futuros profesores de historia en formación. Los resultados muestran una dualidad entre los libros de texto y las narrativas de estudiantes y futuros docentes. Por un lado se aprecian cambios significativos en los manuales escolares que desmitifican dichos contenidos; por otro lado se constata la continuidad tanto entre adolescentes de Secundaria como entre jóvenes docentes en formación de representaciones esencialistas y tradicionales de dichos contenidos fruto de la continuidad de una narrativa maestra.

Desde una perspectiva temporal también amplia, José Ignacio Ortega Cervigón (Universidad Complutense de Educación) y Juan Esteban Rodríguez (Universidad Antonio de Nebrija) estudian cómo han influido los avatares políticos de los últimos cuarenta años en la manera de transmitir la Guerra de la Independencia en los libros de texto. Es un hecho evidente que los manuales de Historia influyen en el imaginario individual del alumno así como en la creación de una memoria colectiva y de unos estereotipos culturales. Pueden contribuir a estigmatizar una época o a un personaje histórico, o bien a ensalzar para siempre la figura de un determinado monarca o grupo social. Partiendo de la convicción de que el libro de texto ha sido el recurso didáctico más utilizado para el desarrollo de la asignatura de Historia, resulta muy interesante prestar atención a la manera en que estos libros han presentado a nuestros escolares la historia, hechos y personajes de la Guerra de la Independencia, 
al ser esta uno de los procesos históricos más utilizados en la creación y consolidación de identidades colectivas.

El artículo de Raimundo A. Rodríguez, M. del Mar Simón y Sebastián Molina (Universidad de Murcia) elige un tema clave imbricado en la construcción de identidades colectivas (el patrimonio regional) en los libros de texto desde la LOGSE (1990) a la LOMCE (2013). Se han analizado las actividades de manuales escolares de las principales editoriales de $5 .^{\circ}$ y $6 .^{\circ}$ de Primaria y algunos libros de Historia de Educación Secundaria Obligatoria. El objetivo ha sido comprobar si, a la par que se forja una narrativa nacional, a través de conceptos, hechos, fechas y personajes de especial relevancia, sucede algo parecido en el ámbito de la narrativa regional. Llama la atención que el escaso espacio dedicado a la actual Región de Murcia aluda a cuestiones culturales o de patrimonio material, en tanto la historia peninsular o universal se centra sobre todo en lo político y, en menor medida, lo social y económico. En cualquier caso, en ambas vertientes (estatal y autonómica) predominan contenidos, actividades e ilustraciones de tipo memorístico y conceptual, que impiden que los alumnos se familiaricen con los hitos, peculiaridades y procesos de cambio y permanencia. Una visión lineal y acrítica de la historia, que en vez de hacer uso del patrimonio para una mejor comprensión del pasado y su puesta en valor, lo emplea como mero adorno erudito, lo cual le aleja de cualquier utilidad ligada al pensamiento histórico, empatía o educación cívica.

Más centrado en un enfoque de análisis de libros de texto desde la actualidad, el artículo de Delfín Ortega (Universidad de Burgos) y Francisco Rodríguez Lestegás (Universidad de Santiago de Compostela) analiza el tratamiento curricular de la Historia de Iberoamérica y su contribución a la construcción de la conciencia histórica iberoamericana en los currículos y libros de texto de Ciencias Sociales españoles para la Educación Primaria. Desde perspectivas inclusivas, interculturales e identitarias, aborda los mecanismos de consolidación y fortalecimiento del Espacio Cultural Iberoamericano en el ámbito educativo, examinando las narrativas históricas e iconográficas escolares, a partir del diseño, validación y aplicación de cinco instrumentos de recogida de datos, análisis y evaluación. Los resultados informan de la sólida adopción de perspectivas eurocéntricas y hegemónicas frente a la ausencia elocuente de una verdadera capacidad integradora de contenidos sociales, culturales e histó- 
ricos orientados a la consolidación del Espacio Cultural Iberoamericano y a la construcción de la reconocida identidad cultural iberoamericana.

Desde una perspectiva similar (análisis de libros actuales sin un enfoque diacrónico) el artículo de Cosme J. Gómez (Universidad de Murcia) y Arthur Chapman (UCL-Institute of Education) examina los enfoques historiográficos y las representaciones sociales, nacionales, europeas y extra-europeas en los libros de texto de Educación Secundaria de España, Francia e Inglaterra. Los resultados muestran diferentes enfoques historiográficos en los manuales analizados, con un peso mayor del estructuralismo y del positivismo en el caso español; una clara influencia de las últimas generaciones de Annales en el caso francés; y un gran peso de la historia social y la historia desde abajo en el caso inglés. Además los tres países presentan diferencias en las representaciones sociales. Los manuales franceses y españoles plantean el relato histórico dentro de un marco europeo del que se sienten partícipes. Sin embargo, los libros de texto ingleses presentan la construcción de la nación inglesa (sobre todo en los siglos de la Edad Media y Edad Moderna) desde una perspectiva muy anglocéntrica. Una circunstancia que tiene unas repercusiones evidentes en la construcción de identidades colectivas a nivel cultural y político, y que ha tenido consecuencias directas en los últimos tiempos.

El dossier, por tanto, presenta un gran interés para el ámbito educativo, para los investigadores en didáctica de la historia, en las representaciones sociales, la historia de la educación y la historia cultural. Los libros de texto son herramientas culturales clave para entender la forma en que se construyen las narrativas maestras de la nación por los poderes públicos y cómo se reproducen en el ámbito educativo. Con demasiada frecuencia la historia ha sido utilizada por el poder para transmitir su mensaje y convertirlo en un discurso socialmente aceptado. La diversidad de enfoques y temáticas del dossier permite analizar estas circunstancias desde múltiples perspectivas y desde una visión poliédrica. ¿Seremos capaces de convertir a los libros de texto de historia en herramientas que permitan una educación histórica basada en una conciencia crítica, en una metodología rigurosa de análisis social y formadora de valores cívicos y ciudadanos? Los resultados de investigación muestran la difícil desconexión de estos manuales escolares con la narrativa impuesta desde las instancias de poder. 


\section{Referencias:}

Álvarez Junco, José, coord. Las historias de España. Visiones del pasado y construcción de identidad. Madrid: Crítica-Marcial Pons, 2013.

- Dioses útiles. Naciones y nacionalismos. Barcelona: Galaxia Gutenberg, 2016.

Armas, Xose M., and Ramón LóPez FACAL. «Ciencias sociales y educación para la ciudadanía. Un diálogo necesario». Íber. Didáctica de las Ciencias Sociales, Geografía e Historia 71 (2012).

AudigIER, François. L'éducation à la citoyenneté. Paris: INRP, 1999.

AudigIER, François, and Nicole TutIAUX-GUILlON. Regards sur l'histoire, la géographie et l'éducation civique à l'école primaire. Lyon: INRP, 2004.

BAGE, Grant. Narrative Matters. Teaching and Learning History through Story. New York: Routledge, 1999.

BARCA, Isabel. O pensamento histórico dos joves. Braga: Universidade do Minho, 2000.

BARTON, Keith C. "Historia e identidad: el reto de los investigadores pedagógicos». In Metodología de la investigación en Didáctica de las Ciencias Sociales. 13-28. Zaragoza: Instituto Fernando el Católico-AUPDCS, 2010.

BARTON, Keith, and Linda LevstiK. Teaching History for the Common Good. New Jersey: Lawrence Erlbau, 2004.

Bauman, Zigmunt. Modernidad líquida. Buenos Aires: Corregidor, 2003.

CARretero, Mario, Alberto Rosa and María Fernanda GonzÁlez. «Enseñar historia en tiempos de memoria». In Enseñanza de la historia y memoria colectiva, edited by Mario Carretero, Alberto Rosa and María Fernanda González, 1136. Buenos Aires: Editorial Paidós, 2006.

CARretero, Mario, and Marcelo Borrelli. "Memoria y enseñanza de la historia en un mundo global. Problemas y desafíos». Íber. Didáctica de las Ciencias Sociales, Geografía e Historia 55 (2008).

CARretero, Mario, and Miriam KRIGER. «¿Forjar patriotas o educar cosmopolitas? El pasado y el presente de la historia escolar en un mundo global». In Aprender y pensar la historia, edited by Mario Carretero and James Voss, 7198. Buenos Aires: Amorrortu, 2004.

CARretero, Mario. "Comprensión y aprendizaje de la historia». In Enseñanza y aprendizaje de la historia en la educación básica. México: Secretaría de Educación Pública, 2011.

Carretero, Mario, José Antonio Castorina, María Sarti, Floor Van Alphen and Alicia BARREIRO. «La construcción del conocimiento histórico». Propuesta Educativa 39 (1) (2013).

Clark, Penney. New possibilities for the past. Shaping history education in Canada. Vancouver \& Toronto: UBC Press, 2011. 
Chapman, Arthur. «Historical interpretations». In Debates in History Teaching, edited by Ian Davies. London-New York: Routledge, 2011.

- «Taking the perspective of the other seriously? Understanding historical argument». Educar em Revista 42 (2011): 95-106.

CHARTIER, Roger. La historia o la lectura del tiempo. Madrid: Gedisa, 2007.

Foster, Stuart J., and Keith A. Crawford, eds. What Shall We Tell the Children? International Perspectives on School History Textbooks. Greenwich, CT: Information Age Publishing, 2006.

GARcía PÉrez, Francisco, and Nicolás DE ALBA. «Educar para la participación ciudadana. Análisis de las dificultades del profesorado a partir de la experiencia del programa Parlamento Joven». In L'educazione alla cittadinanza europea e la formazione degli insegnanti. Un progetto educativo per la «Strategia di Lisbona», edited by Rosa M. Ávila, Beatrice Borghi and Ivo Mattozzi. Bolonia: Pàtron Editore, 2009.

Gómez, Cosme J. «Pensamiento histórico y contenidos disciplinares en los libros de texto. Un análisis exploratorio de la Edad Moderna en 2. ${ }^{\circ}$ de ESO». Ensayos. Revista de la Facultad de Educación de Albacete 29 (1) (2014): 131-158.

Gómez, Cosme J., and Pedro Miralles. «¿Pensar históricamente o memorizar el pasado? La evaluación de los contenidos históricos en la educación obligatoria en España». Revista de Estudios Sociales 52 (2015): 52-68.

- «Los contenidos de ciencias sociales y las capacidades cognitivas en los exámenes de tercer ciclo de Educación Primaria ¿Una evaluación en competencias?». Revista Complutense de Educación 24 (1) (2013).

Gómez, Cosme J., Jorge ORTUÑo and Sebastián MolinA. «Aprender a pensar históricamente. Retos para la historia en el siglo XXI». Tempo e Argumento 6 (11), (2014): 1-25.

Gómez, Cosme J., Raimundo A. Rodríguez and Ana B. Mirete. «Relación entre metodología docente y uso de recursos de innovación en la enseñanza de la historia. Un análisis a través de los recuerdos de los maestros en formación». Clio. History and History Teaching 42 (2016).

Gómez, Cosme J., Ramón Cózar and Pedro Miralles. «La enseñanza de la historia y el análisis de libros de texto. Construcción de identidades y desarrollo de competencias». Ensayos. Revista de la Facultad de Educación de Albacete 29 (1) (2014): 1-25.

Heimberg, Charles. «L'apport de l'histoire à l'éducation à la citoyenneté». In Vivre la democratie, apprende la democratie. Berne: SER, 2005.

- «L'enseignement de l'histoire dans un pays d'immigration: la Suisse». In Pensar históricamente en tiempos de globalización. Santiago de Compostela: USC, 2011. 
HeImberg, Charles. «Pour une éducation aux citoyennetés ouverte sur le monde». L'école du citoyen. Revue Nationale du Réseau CNDP-CRDP pour l'enseignement de l'histoire et de la géographie 7 (1999).

Hervás, Rosa, and Pedro Miralles. «Nuevas formas de enseñar a pensar. El desarrollo del pensamiento crítico en la enseñanza de las ciencias sociales». Íber. Didáctica de las Ciencias Sociales, Geografía e Historia 42 (2004).

HoвsBawm, Eric J. «Introducción: la invención de la tradición». In La invención de la tradición, edited by Eric J. and Terence Rangers, 7-21. Barcelona: Crítica, 2002.

LE GofF, Jacques. Histoire et memoire. Paris: Galimard, 1988.

LÉVEsque, Stephane. Thinking Historically. Educating Students for the 21th Century. Toronto: University of Toronto Press, 2008.

LÓPEZ FACAL, Ramón. «Competencias y enseñanza de las ciencias sociales». Íber. Didáctica de las Ciencias Sociales, Geografía e Historia 74 (2013).

- «Enseñar historia en convivencia plurinacional». Gerónimo de Ustariz 17-18 (2002): 49-57.

— «Identidades posnacionales y enseñanza». Íber. Didáctica de las Ciencias Sociales, Geografía e Historia 47 (2006): 54-63.

- «Nacionalismos y europeísmos en los libros de texto: identificación e identidad nacional». Clío y asociados: la historia enseñada 14 (2010).

Miralles, Pedro. "La didáctica de la historia en España: retos para una educación de la ciudadanía». In L'educazione alla cittadinanza europea e la formazione degli insegnanti. Un progetto educativo per la "Strategia di Lisbona», edited by Rosa M. Ávila, Beatrice Borghi and Ivo Mattozzi. Bologna: Pàtron Editore, 2009.

Miralles, Pedro, and María Begoña Alfageme. «Educación, identidad y ciudadanía en un mundo globalizado y posmoderno». Educatio siglo XXI 31 (1) (2013): 11-12.

Miralles, Pedro, Joaquim Prats and Mercé TATJER. «Conocimientos y concepciones de estudiantes españoles y latinoamericanos de Educación Secundaria Obligatoria sobre las independencias políticas americanas». Scripta Nova. Revista Electrónica de Geografía y Ciencias Sociales XVI (418) (2012).

Miralles, Pedro, Sebastián Molina and Jorge OrTuÑo. La importancia de la historiografía en la enseñanza de la historia. Granada: GEU, 2011.

Monteagudo, José, and José Luis Villa. "La evaluación de las competencias básicas en la materia de Historia de $4 .^{\circ}$ de ESO de la Región de Murcia». In La evaluación en el proceso de enseñanza y aprendizaje de las ciencias sociales, edited by Pedro Miralles Martínez, Sebastián Molina Puche and Antoni Santisteban Fernández. Murcia: AUPDCS, 2011. 
MonTE-SAnO, Chauncey. «Disciplinary literacy in history: An exploration of the historical nature of adolescents' writing». The Journal of the Learning Sciences 19 (4) (2010): 539-568.

Nicholls, Jason. «Methods in School Textbooks Research». International Journal of Historical Learning. Teaching and Research 3 (1) (2003).

PAGÈs, Joan. «Competencia social y ciudadana». Aula de innovación educativa 187 (7) (2009).

PÉREZ-GARZÓN, Juan Sisinio. «¿Por qué enseñamos geografía e historia? ¿Es tarea educativa la construcción de identidades?». Historia de la educación 27 (2008): 37-55.

— «Usos y abusos de la historia». Gerónimo de Ustariz 17-18 (2002): 11-24.

Pinnington, Elisabeth, and David Schugurensky, ed. Learning citizenship by practicing democracy: international initiatives and perspectives. Newcastle upon Tyne: Cambridge Scholarls Publishing, 2010.

PrATs, Joaquim. «En defensa de la historia como materia educativa». Tejuelo. Didáctica de la Lengua y la Literatura 9 (2010).

- «Memoria histórica "versus" historia enseñada». Íber. Didáctica de las Ciencias Sociales, Geografía e Historia 55 (2008).

Prats, Joaquim, and Joan SANTACANA. "¿Por qué y para qué enseñar historia?». In Didáctica de la Geografía y la Historia, edited by Joaquim Prats, 13-29. Barcelona: Graó, 2011.

Ricoeur, Paul. History, Memory, Forgetting. Chicago \& London: University of Chicago Press, 2004.

RodRíguez LeSTEgÁs, Francisco. «La construcción de identidades en la sociedad actual: un desafío para la educación y para la democracia». Revista Española de Pedagogía 239 (2008): 85-102.

RosA, Alberto. "Memoria, historia e identidad. Una reflexión sobre el papel de la enseñanza de la historia en el desarrollo de la ciudadanía». In Aprender y pensar la historia, edited by Mario Carretero and James Voss, 47-70. Madrid: Amorrortu, 2004.

RÜSEN, Jörn. History: Narration, Interpretation, Orientation. New York: Berghahn, 2005.

SÁIz, Jorge. «Educación histórica y narrativa nacional». PhD diss., Universidad de Valencia, 2015.

SCHugurENSKY, David. "Civic engagement and participatory governance». In $\mathrm{Ci}$ vic Engagement: 100th Arizona Town Hall, edited by R. Kelly. Phoenix: Arizona Town Hall, 2012.

SEIXAS, Peter. "The Community of Inquiry as a Basis for Knowledge and Learning: The Case of History». American Educational Research Journal 30 (2) (1993). 
STARKEY, Hugh. "Citizenship, education and global spaces». Language and Intercultural Communciation 11 (2) (2011).

- «Human Rights and Education». In Encyclopedia of Diversity in Education, edited by James Banks. London: SAGE, 2012.

THÉNARD-DuVIVIER, Franck, coord. L'enseignement en histoire et géographie. Paris: ADAPT, 2008.

THORNTON, Stephen J. "What is history in US history textbooks?». In School history textbooks across cultures: International debates and perspectives, edited by Jason Nicholls. Oxford, UK: Symposiumbooks, 2006.

ToPOLSKY, Jerzy. "La estructura de las narrativas históricas y la enseñanza de la historia». In Aprender y pensar la historia, edited by Mario Carretero and James Voss, 101-120. Buenos Aires: Amorrortu, 2004.

Valls, Rafael. La enseñanza de la Historia y textos escolares. Buenos Aires: Zorzal, 2008.

VANSLEDRIGHT, Bruce. "Narratives of Nation-State, Historical Knowledge and School History Education». Review of Research in Education 32 (1) (2008): 109-146.

- The Challenge of Rethinking History Education. On Practice, Theories, and Policy. New York: Routledge, 2011. 